\title{
Tuning the Kosterlitz-Thouless transition to zero temperature in anisotropic boson systems
}

\author{
Jhih-Shih You, ${ }^{1,2}$ Hao Lee, ${ }^{1,2}$ Shiang Fang, ${ }^{1,2}$ Miguel A. Cazalilla, ${ }^{3,4,5}$ and Daw-Wei Wang ${ }^{1,2}$ \\ ${ }^{1}$ Physics Department and Frontier Research Center on Fundamental and Applied Sciences of Matter, National Tsing-Hua University, \\ Hsinchu, Taiwan \\ ${ }^{2}$ Physics Division, National Center for Theoretical Sciences, Hsinchu, Taiwan \\ ${ }^{3}$ Centro de Fsica de Materiales CSIC-UPVIEHU, Paseo Manuel de Lardizabal 5, E-20018 San Sebastian, Spain \\ ${ }^{4}$ DIPC, Paseo Manuel de Lardizabal 4, E-20018 San Sebastian, Spain \\ ${ }^{5}$ Graphene Research Centre National University of Singapore, 6 Science Drive 2, Singapore 117546
}

(Received 22 July 2012; published 10 October 2012)

\begin{abstract}
We study the two-dimensional Bose-Hubbard model with anisotropic hopping. Focusing on the effects of anisotropy on superfluid properties such as the helicity modulus and the normal-to-superfluid [BerezinskiiKosterlitz-Thouless (BKT)] transition temperature, two different approaches are compared: large-scale quantum Monte Carlo simulations and the self-consistent harmonic approximation (SCHA). For the latter, two different formulations are considered, one applying near the isotropic limit and the other applying in the extremely anisotropic limit. Thus we find that the SCHA provides a reasonable description of superfluid properties of this system provided the appropriate type of formulation is employed. The accuracy of the SCHA in the extremely anisotropic limit, where the BKT transition temperature is tuned to zero (i.e., at a quantum critical point) and therefore quantum fluctuations play a dominant role, is particularly striking.
\end{abstract}

DOI: 10.1103/PhysRevA.86.043612 PACS number(s): 03.75.Lm, 05.30.Jp, 05.70.Jk, 71.10.Pm

\section{INTRODUCTION}

In recent years, much progress has been made in ultracold atoms loaded in optical lattices [1-3]. Several experimental groups have demonstrated the great tunability of such systems by driving them from a superfluid to a Mott insulator phase (and vice versa) in various dimensions and lattice geometries [1,4-9]. By varying the laser intensity along one or several directions, experimentalists can control the hopping anisotropy for the atoms in the optical lattice $[4,5,8,10]$. Thus, some of these experiments have started to explore the fascinating behavior of ultracold atoms confined to low dimensions $[4,5,8,10]$. This control makes it possible to study dimensional crossovers also [4,11-14] as well as a wide range of other phenomena [4,8], which are also relevant for the understanding of complex solid-state systems such as layered superconductors [15-18] and anisotropic magnetic materials [19-22].

Indeed, matter in low dimensions is known to display a wide range of exotic properties, which are hard to find in three-dimensional systems. These include fractionalization of quantum numbers [23], critical states lacking long-range order $[4,8,10,24]$, and topological phase transitions that cannot be characterized by an order parameter, such as the Berezinskii-Kosterlitz-Thouless (BKT) transition [10,25,26]. The question of how these exotic properties evolve as lowdimensional systems are coupled and become, by virtue of the coupling, higher-dimensional systems has attracted a great deal of experimental and theoretical attention in recent years $[4,11-14,21,22,24,27-29]$.

In bosonic systems, such as ultracold gases of bosonic atoms or molecules, as well as in anisotropic magnetic materials [24], a theoretical analysis of the dimensional crossovers and other interesting phenomena such as deconfinement transitions [13,14] can be carried out through a combination of perturbative renormalization-group (RG) and mean-field theory (MFT) approaches. MFT assumes the existence of a
Bose-Einstein condensate and it is expected to be a reliable description of the anisotropic superfluid phase only if the crossover takes place from one to three dimensions. However, when trying to describe the crossover from one (1D) to two dimensions (2D) at finite temperatures, MFT breaks down because bosons in two dimensions fail to condense at all temperatures except at $T=0$. Nevertheless, under such conditions a qualitative understanding of the properties of the anisotropic superfluid phase can still be obtained by means of perturbative RG and variational methods, as we shall demonstrate below. However, an independent check of these approximated methods is still required.

It is worth noting that the superfluid properties appear to be strongly dependent on the system dimensionality [26,30-34]. Experimentally, a superfluid response has been observed at finite temperatures in both two- [26] and one-dimensional [30] interacting boson systems, which lack a Bose-Einstein condensate. However, the origin of the superfluidity in these two cases is very different [31,34]: Whereas in 2D the superfluid response is essentially a thermodynamic phenomenon that is quantified by the helicity modulus [31], in 1D it is a dynamic property as the helicity modulus vanishes at all temperatures (the helicity modulus at zero temperature is obtained by taking the $T \rightarrow 0$ limit after taking the thermodynamic limit [32,34]). The vanishing helicity modulus of the 1D Bose fluid is in stark contrast with the finite helicity modulus of a 2D superfluid, which changes discontinuously across the BKT transition. As will be discussed below, making the hopping amplitude in one direction vanishingly small drives the BKT transition temperature to the absolute zero (at $T=0$ ) and the transition thus becomes a 2D $X Y$ quantum critical point $(\mathrm{QCP})$ at the end of a line of classical 2D $X Y$ critical points (see Fig. 1). The line of classical 2D $X Y$ points separates the anisotropic normal and superfluid phases. Furthermore, at the QCP two other critical lines also meet: a $1 \mathrm{D} X Y$ line corresponding to $t_{y} / t_{x}=0$ and a 3D $X Y$ line corresponding to $T=0$ and finite $t_{y} / t_{x}$. Therefore, 


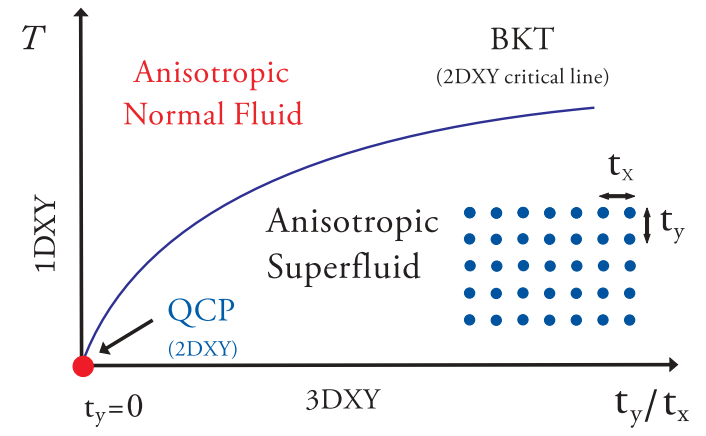

FIG. 1. (Color online) Schematic phase diagram for the BoseHubbard model with hopping anisotropy ratio in two dimensions.

it can be argued that the helicity modulus vanishes in 1D Bose fluids because these fluids exhibit the same superfluid response as the normal phase of the anisotropic 2D fluid in the limit of vanishing anisotropy ratio. This can be seen by noticing that, in order to compute the helicity modulus of a 1D Bose fluid, we can approach the 1D $X Y$ line at a finite temperature $T>0$ from the normal-fluid phase by taking $t_{y} / t_{x} \rightarrow 0$ (see Fig. 1). On the other hand, reaching the 1D $X Y$ line from the 2D (anisotropic) superfluid phase is not possible without going through a (classical or quantum) critical point, which is a thermodynamic singularity (see Fig 1).

Indeed, the variety of phenomena that can be studied in anisotropic bosonic systems is very wide [13,14,24]. In this work, we focus on understanding the properties of the anisotropic superfluid phase that can be realized in, e.g., two-dimensional optical lattices with hopping anisotropy. However, our results can also be of relevance to much more complex solid-state systems, such as anisotropic magnetic insulators [21,22,24]. In particular, we are interested in understanding how the hopping anisotropy affects the properties of the superfluid phase (i.e., the helicity modulus) and the Berezinskii-Kosterlitz-Thouless transition temperature from the superfluid to the normal-fluid phase. As the BKT transition temperature is tuned towards $T=0$ by the hopping anisotropy, the importance of quantum fluctuations is enhanced. Thus, we expect that this will lead to important renormalization effects on the parameters of the effective 2D $X Y$ model that describes the line of classical critical points. Below we shall rely on the self-consistent harmonic approximation (SCHA) to various limits of the quantum rotor model to estimate such renormalization effects. The results of the calculations based on the SCHA for the critical temperature and the helicity modulus will be compared with quantum Monte Carlo simulations.

Of course, the effect of quantum fluctuations is enhanced not only by the anisotropy but also by the interparticle interaction, which can drive a quantum phase transition from the superfluid phase to a Mott-insulator phase at integer fillings. In various dimensions, such a superfluid-to-Mottinsulator transition has been extensively studied both experimentally and theoretically, mainly in isotropic systems [1,4-9]. However, the combined effect of anisotropy and interparticle interactions in enhancing the quantum fluctuations and destroying superfluidity in two-dimensional Bose systems has

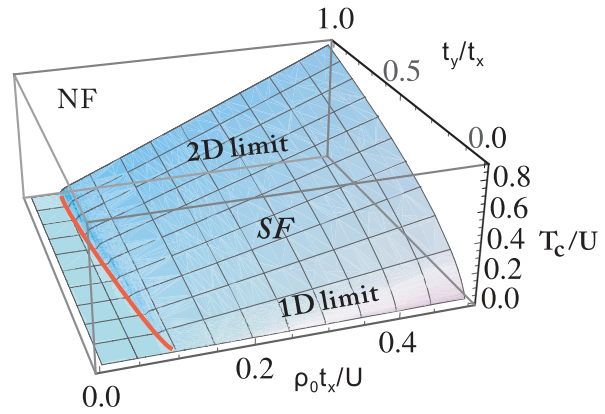

FIG. 2. (Color online) The complete phase diagram of an anisotropic Hubbard model as a function of anisotropy ratio $t_{y} / t_{x}$, interaction strength $\rho_{0} t_{\alpha} / U$, and temperature $T / U$. Note that we keep $U$ constant and vary other quantities for convenience. This diagram is calculated by the SCHA as described in the text. The red line guides zero-temperature transitions (from the superfluid to the Mott-insulator phase). SF indicates the superfluid and NF the normal fluid.

not been studied so far. In this paper, we shall show how both quantum and thermal fluctuations can be treated on equal footing in the study of an anisotropic superfluid in a 2D optical lattice. Our results can be summarized in the phase diagram shown in Fig. 2.

The outline of this article is as follows: In Sec. II we introduce the relevant low-energy models that we use to describe the anisotropic Bose-Hubbard model (BHM) in 2D. Several analytic and numerical approximations to the BHM are also discussed there. We also discuss the problem of how to estimate the "phase-stiffness" parameters of the $2 \mathrm{D} X Y$ model that describes the critical line of BKT transitions separating the normal and superfluid phases at finite temperature (cf. Figs. 1 and 2). In Sec. III, the effects of thermal fluctuations and interaction on the helicity modulus from small to intermediate anisotropy are discussed. In Sec. IV we explore these renormalization effects in the extremely anisotropic regime, as well as the behavior of the BKT critical temperature. The conclusions of this work can be found in Sec. V. The Appendixes contain the technical details of the SCHA calculations in the various limits of the quantum rotor model.

\section{MODELS AND METHODS}

Anisotropy of single-particle tunneling can be easily realized in an optical lattice by using different laser intensities for the standing waves in the $x$ and $y$ directions. In the limit of a deep lattice, in which essentially all particles reside in the lowest Bloch band, the system can be described by the single-band Bose-Hubbard model:

$$
H=-\sum_{\langle i, j\rangle} t_{i j} b_{i}^{\dagger} b_{j}-\mu \sum_{i} \hat{n}_{i},+\frac{U}{2} \sum_{i} n_{i}\left(n_{i}-1\right),
$$

where $b_{i}^{\dagger}$ is the bosonic creation operator on site $\mathbf{R}_{i}$, and $n_{i}=$ $b_{i}^{\dagger} b_{i}$ is the boson occupation operator. The tunneling amplitude is $t_{i j}=t_{x}\left(t_{i j}=t_{y}\right)$, if $\mathbf{R}_{i}-\mathbf{R}_{j}= \pm a \hat{\mathbf{x}}\left(\mathbf{R}_{i}-\mathbf{R}_{j}= \pm a \hat{\mathbf{y}}\right)$ with $a$ being the lattice constant. $U$ and $\mu$ are the on-site interaction and chemical potential, respectively. 


\section{A. The $X Y$ model and the self-consistent harmonic approximation}

Deep into the superfluid phase, the low-temperature behavior of the system is largely dominated by phase fluctuations. For sufficiently large values of $U$ and for the bare anisotropy ratio parameter $\eta_{0}=J_{y}^{0} / J_{x}^{0} \approx 1$, we can represent the boson operator as $b_{i}=\sqrt{\rho_{0}+\delta \rho_{i}} e^{\mathrm{i} \theta_{i}}$, where $\rho_{0}$ is the mean boson occupation, while $\theta_{i}$ and $\delta \rho_{i}\left(\ll \rho_{0}\right)$ describe the phase and density fluctuations at site $\mathbf{R}_{i}$, respectively. After integrating out the density fluctuations, the partition function $(Z)$ of the system can be written as a (Feynman) functional integral, $Z=\int \mathcal{D} \theta e^{-S_{X Y}[\theta]}$, where $(\hbar=1)$

$$
S_{X Y}[\theta]=\int_{0}^{\beta} d \tau\left[\sum_{i} \frac{\left(\partial_{\tau} \theta_{i}\right)^{2}}{2 U}-\sum_{\langle i, j\rangle} 2 J_{i j}^{0} \cos \left(\theta_{i}-\theta_{j}\right)\right]
$$

is the two-dimensional $\mathrm{O}(2)$ quantum rotor model, with $J_{i j}^{0}=\rho_{0} t_{i j}$ being the bare Josephson coupling and $\beta=1 / T$ the inverse of the absolute temperature in units where the Boltzmann constant $k_{B}=1$.

At sufficiently high temperatures, the imaginary-time $(\tau)$ dependence of the phase $\theta_{i}$ can be neglected and the model in Eq. (2) becomes the classical ferromagnetic $X Y$ model:

$$
S_{\mathrm{C} X Y}[\theta]=-2 \beta \sum_{\langle i, j\rangle} J_{i j}^{0} \cos \left(\theta_{i}-\theta_{j}\right)
$$

In 2D this model has two distinct phases: In the hightemperature regime, the orientation of the rotors described by the phase $\theta_{i}$ is disordered. The phase correlations are short ranged, i.e., $g_{i j}=\left\langle e^{i \theta_{i}} e^{-i \theta_{j}}\right\rangle \simeq e^{-\left|\mathbf{R}_{i}-\mathbf{R}_{j}\right| / \xi(T)}$, where the correlation length $\xi(T) \gg a$. Such behavior corresponds to a normal phase. On the other hand, in the low-temperature regime, the phase correlations decay algebraically, i.e., $g_{i j} \simeq$ $\left|\mathbf{R}_{i}-\mathbf{R}_{j}\right|^{-\alpha(T)}$, where the exponent $\alpha(T)$ is finite and related to the thermodynamic phase stiffness. This behavior corresponds to a superfluid phase exhibiting quasi-long-range order. The latter implies the absence of a Bose-Einstein condensate, but the finite phase stiffness $\left(J_{i j}^{0}\right)$ means that the system can sustain superflows at all temperatures below the BerezinskiiKosterlitz-Thouless temperature $T_{c}$. Above such temperature, vortices and antivortices unbind and destroy the superfluid properties of the system. The vortices (antivortices) are singular configurations of the phase $\theta_{i}$, where the latter winds out by positive (negative) integer multiples of $2 \pi$ around a discrete set of points on the plane.

The picture described above relies on the classical (hightemperature) limit of the quantum rotor model, where the first term in Eq. (2) $\left[\propto\left(\partial_{\tau} \theta_{i}\right)^{2}\right]$ is neglected. In other words, if we expand

$$
\theta_{i}(\tau)=\frac{1}{\beta} \sum_{\omega_{n}} e^{-i \omega_{n} \tau} \theta_{i}\left(\omega_{n}\right)
$$

where $\omega_{n}=\frac{2 \pi}{\beta} n$ ( $n$ being an integer), the high-temperature limit takes into account the fluctuations of the $\theta_{i}\left(\omega_{n}\right)$ field only for $\omega_{n}=0$. However, the model in Eq. (2) is quantum mechanical, and the quantum fluctuations are described by the finite-Matsubara-frequency (i.e., $\omega_{n} \neq 0$ ) components of $\theta_{i}\left(\omega_{n}\right)$. The latter and the classical (i.e., thermal) configurations described by $\theta_{i}\left(\omega_{n}=0\right)$ are coupled nonlinearly through the Josephson coupling term proportional to $J_{i j}^{0} \cos \left[\theta_{i}(\tau)-\right.$ $\left.\theta_{j}(\tau)\right]$. At low temperatures, both quantum and classical fluctuations must be taken into account. This means that we must obtain the effective classical limit of the quantum rotor model by integrating out the quantum fluctuations described by the $\theta_{i}\left(\omega_{n} \neq 0\right)$ components of the phase. This is especially important for the anisotropic $X Y$ model because, as we drive the system towards the extremely anisotropic limit where $t_{y} / t_{x} \ll 1$, the BKT transition temperature $T_{c}$ tends to zero (cf. Figs. 1 and 2).

In order to carry out the integration of the quantum fluctuations, we shall rely upon the self-consistent harmonic approximation $[13,35]$. Thus, we shall assume that, below $T_{c}$, the quantum rotor model of Eq. (2) can be approximated by an anisotropic Gaussian model:

$$
S_{G}[\theta]=\int_{0}^{\beta} d \tau\left[\sum_{i} \frac{\left(\partial_{\tau} \theta_{i}\right)^{2}}{2 U}+\sum_{\langle i, j\rangle} J_{i j}\left(\theta_{i}-\theta_{j}\right)^{2}\right]
$$

where $J_{i j}$ is the effective Josephson coupling renormalized by the interactions and the thermal fluctuations. The derivation of a self-consistent equation for $J_{i j}$ is given in Appendix A.

\section{B. Josephson-coupled Tomonaga-Luttinger liquids and the SCHA}

For small values of the anisotropy ratio (i.e., for $t_{y} / t_{x} \rightarrow$ 0 ), it is convenient to consider a different limit of the the anisotropic Bose-Hubbard model introduced in Eq. (1). Indeed, for $t_{y}=0$, Eq. (1) reduces to an array of uncoupled 1D Bose gases. For temperatures $T \ll t_{x}$, an interacting 1D Bose gas is known to behave as a Tomonaga-Luttinger liquid (TLL) [24]. Upon restoring a small $t_{y}\left(\ll t_{x}\right)$ coupling between the TLLs, the resulting system is an array of weakly coupled TLLs (CTLLs), which is described by the following effective Hamiltonian [14,24]:

$$
\begin{aligned}
H_{\mathrm{CTLL}}= & \frac{v}{2 \pi} \sum_{i=1}^{L_{y}} \int d x\left[K\left(\partial_{x} \theta_{i}\right)^{2}+K^{-1}\left(\partial_{x} \phi_{i}\right)^{2}\right] \\
& -\frac{g_{J}^{0} v}{\pi a_{0}^{2}} \sum_{i=1}^{L_{y}} \int d x \cos \left[\theta_{i}-\theta_{i+1}\right],
\end{aligned}
$$

where $v$ is the sound velocity, $K$ is the Luttinger parameter characterizing the decay of correlations, $a_{0} \approx a$ is a shortrange cutoff, and $g_{J}^{0} \simeq 2 \pi t_{y} \rho_{0} a_{0}^{2} / v$. The fields $\frac{1}{\pi} \partial_{x} \phi_{i}(x)$ and $\theta_{i}(x)$ describe the (long-wavelength) density and phase fluctuations of the $1 \mathrm{D}$ interacting Bose gas at site $i=1, \ldots, L_{y}$ of the array.

In order to obtain a phase-only description, we integrate out the density fields $\phi_{i}(x)$ in Eq. (6) and thus obtain the following action for the array of weakly coupled TLLs:

$$
\begin{aligned}
S_{\mathrm{CTLL}}\left[\theta_{i}\right]= & \frac{K}{2 \pi} \sum_{i=1}^{L_{y}} \int_{0}^{\beta} d \tau \int_{0}^{L_{x}} d x\left[\frac{\left(\partial_{\tau} \theta_{i}\right)^{2}}{v}+v\left(\partial_{x} \theta_{i}\right)^{2}\right] \\
& -\frac{g_{J}^{0} v}{\pi a_{0}^{2}} \sum_{i=1}^{L_{y}} \int_{0}^{\beta} d \tau \int_{0}^{L_{x}} d x \cos \left(\theta_{i}-\theta_{i+1}\right) .
\end{aligned}
$$


It is now possible to apply the SCHA to this model by approximating the nonlinear Josephson coupling in $S_{\mathrm{TLL}}\left[\theta_{i}\right]$ by a Gaussian coupling:

$$
\begin{aligned}
S_{G}\left[\theta_{i}\right]= & \frac{K}{2 \pi} \sum_{i=1}^{L_{y}} \int_{0}^{\beta} d \tau \int_{0}^{L_{x}} d x\left[\frac{\left(\partial_{\tau} \theta_{i}\right)^{2}}{v}+v\left(\partial_{x} \theta_{i}\right)^{2}\right] \\
& +\frac{g_{J} v}{\pi a_{0}^{2}} \sum_{i=1}^{L_{y}} \int_{0}^{\beta} d \tau \int_{0}^{L_{x}} d x\left(\theta_{i}-\theta_{i+1}\right)^{2},
\end{aligned}
$$

where $g_{J}$ is the effective SCHA coupling. It can be computed by solving the equation in Appendix B.

\section{BKT transition and the sine-Gordon model}

The advantage of the Gaussian models [either (5) or (8)], obtained after the application of the SCHA, is that they allow for readily integrating out the "quantum components" of the phase field (i.e., the $\omega_{n} \neq 0$ components of $\theta$ ). We can thus obtain, in the continuum limit where the variation of the phase is slow over the scale of the lattice parameter, a classical Gaussian (CG) model

$$
S_{\mathrm{CG}}[\theta]=\frac{1}{2} \int d x d y\left[K_{x}\left(\partial_{x} \theta\right)^{2}+K_{y}\left(\partial_{y} \theta\right)^{2}\right],
$$

where the expressions for the stiffnesses $K_{x}$ and $K_{y}$ depend on the starting Gaussian model: $K_{x}=\beta J_{x}$ and $K_{y}=\beta J_{y}$, for Eq. (5), and $K_{x}=\beta K v /(a \pi)$ and $K_{y}=\beta g_{J} /(a \pi)$, for Eq. (8). Interestingly enough, the role of the anisotropy in the continuum-limit description based on (9) seems to be rather minor. This can be seen by rescaling the coordinates $x \rightarrow \eta^{1 / 2} x$ and $y \rightarrow \eta^{-1 / 2} y$, where $\eta=\sqrt{K_{x} / K_{y}}$, yielding the following isotropic Gaussian model:

$$
S_{\mathrm{CG}}[\theta]=\frac{K_{\beta}}{2} \int d \mathbf{r}(\nabla \theta)^{2},
$$

where $K_{\beta}=\sqrt{K_{x} K_{y}}$. Note that, in a finite system, the rescaling also affects the system dimensions: $L_{x} \rightarrow L_{x} \eta^{1 / 2}$ and $L_{y} \rightarrow L_{y} \eta^{-1 / 2}$. This observation will be important below.

Equation (10) can be regarded as the naive continuum limit of Eq. (3) and it can describe the (thermal) phase fluctuations only within the superfluid phase of Eq. (1). Thus, this model can only capture the algebraically decaying phase correlations characterizing the superfluid phase of the $X Y$ model (cf. Sec. II A). However, it is completely unable to capture the vortex and antivortex unwinding that ultimately drives the BKT transition.

In order to capture the possibility of topological excitations that ultimately lead to the BKT transition, we need to take a step back to the original $X Y$ model, either Eq. (2) or Eq. (7), and acknowledge that by relying on the SCHA, since we have neglected the possibility of topological configurations of the phase where the latter jumps by multiples of $2 \pi$ from a given lattice site to a neighboring site. Thus, the right way to proceed would have been to start from the quantum $X Y$ model [or better, from the Bose-Hubbard model of Eq. (1)] and, after integrating out the quantum components of the phase (and density) fields, to arrive at an effective classical $X Y$ model like Eq. (3), with properly renormalized parameters. The latter, via a duality transformation [36,37], can be mapped onto the sine-Gordon
(sG) model,

$$
S_{\mathrm{SG}}=\int d \mathbf{r}\left\{\frac{[\nabla \phi(\mathbf{r})]^{2}}{2 K_{\beta}^{(0)}}-\frac{2 g^{(0)}}{a^{2}} \cos 2 \pi \phi(\mathbf{r})\right\},
$$

where $\phi(\mathbf{r})$ is a field that is dual [36,37] to $\theta(\mathbf{r})$ and $g^{0} \propto$ $e^{-E_{c} / k_{B} T}$ is the so-called vortex fugacity with $E_{c}$ being the vortex core energy. The classical 2D $X Y$ and the sine-Gordon models belong to the same universality class, which means that, near the BKT transition, they provide an equally accurate description of the long-wavelength phenomena. For the 2D $X Y$ universality class, Nelson and Kosterlitz have shown [31] using the renormalization group that, at the critical temperature for the BKT transition, $T_{c}$, the renormalized phase stiffness $\left(K_{\beta}^{(R)}\right)$ exhibits a universal jump:

$$
\begin{gathered}
K_{\beta}^{(R)}\left(T \rightarrow T_{c}^{-}\right)=\frac{2}{\pi}, \\
K_{\beta}^{(R)}\left(T \rightarrow T_{c}^{+}\right)=0 .
\end{gathered}
$$

The renormalized stiffness $K_{\beta}^{(R)}$ satisfies a set of differential RG equations, which describe the 'flow' of the sine-Gordon parameters (which correspond to $K_{\beta}^{(0)}$ and $g^{(0)}$ at the scale of the lattice parameter $a$ ) as the system classical degrees of freedom are coarse grained in the vicinity of the BKT transition. Thus, the RG equations determine the long-wavelength properties of the system, or, in other words, the phase of system: For $K_{\beta}^{(R)}>2 / \pi$ (i.e., for $T<T_{c}$ ), the coupling of the nonlinear term $(\propto \cos 2 \pi \phi)$ in Eq. (11), which is responsible for the creation of vortex-antivortex pairs, is renormalized down to zero, leading us back to the Gaussian model [cf. Eq. (10)] that describes the superfluid phase, but with a renormalized value of the stiffness equal to $K_{\beta}^{(R)}$. On the other hand, when $K_{\beta}^{(R)}<2 / \pi$ (for $T>T_{c}$ ), the vortex-antivortex pairs unbind, which means that the coefficient of the $\cos 2 \pi \phi$ term grows as the system is coarse grained. The unbinding disorders the system, thus destroying the superfluidity (i.e., $K_{\beta}^{(R)} \rightarrow 0$ ), and thus the system becomes a normal Bose fluid.

However, it must be pointed out that the derivation of the sine-Gordon model from the original Bose-Hubbard model [cf. Eq. (1)] or the quantum $X Y$ model, Eq. (2), is very hard to carry out in practice. The reason is that the integration of the $\omega_{n} \neq 0$ components of the phase cannot be performed exactly due to the nonlinear nature of the Josephson coupling. Thus, in this work we have chosen an alternative route, which involves using the SCHA to obtain the Gaussian model with effective parameters $K_{x}$ and $K_{y}$, from which we can obtain an approximation to the renormalized stiffness at $T_{c}: K_{\beta}^{R}\left(T_{c}\right) \approx K_{\beta}\left(T_{c}\right)=\sqrt{K_{x}\left(T_{c}\right) K_{y}\left(T_{c}\right)}$. As we shall show below by explicit comparison with quantum Monte Carlo (QMC) results, the SCHA provides a reasonably accurate estimate of the superfluid parameters even in an anisotropic Bose system where $T_{c}$ is driven to zero. Within this framework, an approximation to the critical temperature is determined from the condition that

$$
K_{\beta}\left(T_{c}\right)=\frac{2}{\pi}
$$


Note that, since $K_{\beta}$ is not the actual renormalized stiffness, it does not necessarily vanish for $T>T_{c}$. However, in accordance with (13) we impose this fact by hand.

\section{QMC simulation on the Bose-Hubbard model}

In order to validate the previously described approximations, we have carried out $a b$ initio QMC simulations of the Bose-Hubbard model Eq. (1) using the worm algorithm [38]. Earlier work $[39,40]$ on isotropic 2D interacting Bose systems has shown that this algorithm can be used to study the KT transition. However, as pointed out by Prokof'ev and Svistunov in Ref. [41], the helicity modulus depends strongly on the aspect ratio of the lattice employed in the QMC simulation, i.e., when the thermodynamic limit $\left(L_{x, y} \rightarrow \infty\right)$ is taken by keeping $L_{x} / L_{y}$ fixed in isotropic systems where $t_{x}=t_{y}$. As a result, the definition of superfluidity and its transition temperature can be different for different aspect ratios. The reason is that, as $L_{x} / L_{y}$ is varied away from unity, the criticality of the system also undergoes a crossover from a classical 2D $X Y$ to a $1 \mathrm{D} X Y$ universality class. In the latter case, $T_{c}$ and the helicity modulus vanish. The crossover would be complete if we were able to conduct simulations up to the thermodynamic limit. However, in finite systems finite-size effects prevent the system from completely reaching the 1D $X Y$ fixed point.

In this work, we focus on the effect of the hopping anisotropy, $\eta_{0}=t_{y} / t_{y} \neq 1$, on the superfluid properties. Therefore, we must first determine a physically sensible prescription to obtain the helicity modulus and hence the BKT transition temperature. To this end, in our QMC simulations we have chosen a value of the system aspect ratio $L_{x} / L_{y}$ such that the excitation energy of a unit quantized flux is the same in both directions in the noninteracting limit, i.e., $t_{x}\left(\frac{2 \pi}{L_{x}}\right)^{2}=t_{y}\left(\frac{2 \pi}{L_{y}}\right)^{2}$ or $L_{x} / L_{y}=\sqrt{t_{x} / t_{y}}$. For example, for $t_{y} / t_{x}=0.1$, we use $L_{x}=100$ and $L_{y}=32$ so that $L_{x} / L_{y}=3.125 \simeq \sqrt{t_{x} / t_{y}}=$ $\sqrt{10}=3.1622$. The rationale for this choice is explained in what follows.

The helicity modulus can be defined as $[42,43]$

$$
\gamma_{x, y}=\frac{2 \Delta F\left(\phi_{x, y}\right)}{\Omega\left(\phi_{x, y} / L_{x, y}\right)^{2}},
$$

where $\Omega=L_{x} L_{y}$ is the system area and $\Delta F\left(\phi_{x, y}\right)$ is the free-energy change due to an infinitesimal phase twist $\phi_{x, y}$ applied at the boundaries of the system. However, in a QMC simulation, the helicity modulus can be also obtained from the winding-number fluctuations $\left\langle W_{x, y}^{2}\right\rangle[41,43]$ :

$$
\gamma_{x, y}=T \frac{L_{x, y}}{L_{y, x}}\left\langle W_{x, y}^{2}\right\rangle,
$$

where $\left\langle W_{x}^{2}\right\rangle\left(\left\langle W_{y}^{2}\right\rangle\right)$ are the winding-number fluctuations along the $x(y)$ direction.

In continuum systems, the helicity modulus $\gamma$ can be related to a quantity with dimensions of density, namely, the superfluid density $\rho_{s}$, by means of the equation

$$
\gamma=\frac{h^{2}}{m} \rho_{s}
$$
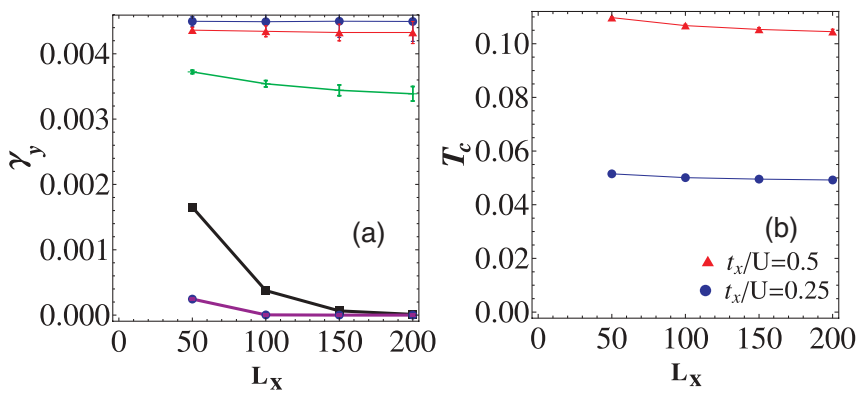

FIG. 3. (Color online) (a) The helicity modulus in the $y$ direction, $\gamma_{y}$, obtained by QMC simulations as a function of the longest side $L_{x}$. We use $\eta_{0}=J_{y}^{0} / J_{x}^{0}=0.02, t_{x} / U=0.25$, and $\rho_{0} \simeq 0.8$. From top to bottom the lines are for $T / U=0.01$ to 0.09 in steps of 0.02 . (b) $T_{c}$ as a function of $L_{x}$ for $\eta_{0}=J_{y}^{0} / J_{x}^{0}=0.02 . t_{x} / U=0.5, \rho_{0} \simeq$ 0.7 for red triangles and $t_{x} / U=0.25, \rho_{0} \simeq 0.8$ for blue dots.

where $m$ is the particle mass. In lattice systems, a natural generalization of (17) is obtained by replacing $m$ by the effective mass $m_{x, y}^{*}$, which may be direction dependent. Indeed, for free particles, $\frac{\hbar^{2}}{m_{x, y}^{*}} \sim 2 t_{x, y}$, which implies that

$$
\gamma_{x, y}=2 t_{x, y} \rho_{s} .
$$

Therefore, the choice of aspect ratio $L_{x} / L_{y}=\sqrt{t_{x} / t_{y}}$ means [cf. Eq. (16)] that $\left\langle W_{x}^{2}\right\rangle=\left\langle W_{y}^{2}\right\rangle$ and thus the superfluid density $\rho_{s}$ alone determines the helicity modulus in both directions.

Next, let us assess the importance of finite-size effects using the above choice for the system aspect ratio. In Fig. 3(a), we show the helicity modulus in the $y$ direction, $\gamma_{y}$, as a function of the longest side $L_{x}$, where $t_{x} / U=0.25, \rho_{0} \simeq 0.8$, and $\eta_{0}=J_{y}^{0} / J_{x}^{0}=0.02$. We find that, for $L_{x} \geqslant 100$ and $T \leqslant 0.05$, $\gamma_{y}$ is almost unchanged by the variation of $T / U$ from 0.01 to 0.09 . In Fig. 3(b), we show $T_{c}$ as a function of $L_{x}$ for $\eta_{0}=J_{y}^{0} / J_{x}^{0}=0.02$. It can be seen that the variation of $T_{c}$ (see Sec. III B for an explanation of how $T_{c}$ is estimated from the QMC data) with $L_{x}$ is less than $5 \%$. These results justify the neglect of finite-size effects on the helicity modulus and $T_{c}$ for the typical system sizes employed in our QMC simulations $\left(L_{x}>100\right)$.

\section{SMALL TO INTERMEDIATE ANISOTROPY}

\section{A. Inside the superfluid phase}

We first discuss the results of the SCHA for the $X Y$ model, which approximates Eq. (2) by the Gaussian model of Eq. (5) with an effective quadratic coupling $J_{i j}\left(T, U, n_{0}\right)$. The derivation of the equation for $J_{i j}$ is given in Appendix A [cf. Eq. (A12)]. We note that the nonlinear Josephson term in Eq. (2) couples all Matsubara frequencies, and therefore, in the SCHA, the renormalized $J_{i j}$ in Eq. (5) acquires a temperature dependence.

From the continuum limit of the Gaussian model obtained from the SCHA [cf. Eq. (5)], the helicity modulus can be read off: $\gamma_{x, y}=2 J_{x, y}$. Hence, we can also define the anisotropy ratio as $\eta=\gamma_{y} / \gamma_{x}=J_{y} / J_{x}$. For later purposes, it is also worth introducing the bare (i.e., unrenormalized) system parameters: $\gamma_{x, y}^{0}=2 J_{x, y}^{0}=2 t_{x, y} \rho_{0} \quad\left(\rho_{0}\right.$ is the mean lattice occupation and $a$ the lattice parameter) and the bare anisotropy ratio $\eta_{0}=\gamma_{y}^{0} / \gamma_{x}^{0}=J_{y}^{0} / J_{x}^{0}=t_{y} / t_{x}$. In what follows, we compare 

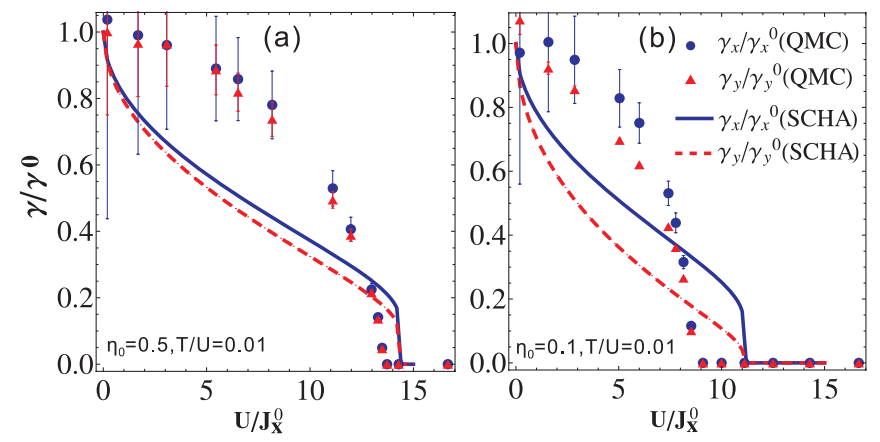

FIG. 4. (Color online) The renormalized helicity modulus $\gamma_{\alpha} / \gamma_{\alpha}^{0}$ as a function of the interaction strength $U / J_{x}^{0}$. Note that, on both figures, we keep constant the values of $U, T$, and $\eta_{0}=J_{y}^{0} / J_{x}^{0}=0.5$ and 0.1 , and change $J_{x}^{0}$. Blue solid (red dashed) lines represent the results in the $\alpha=x(y)$ directions. For comparison, we also show the numerical results obtained by QMC simulations in filled circles and triangles. See the text for more details of the comparison.

the results of $\gamma_{x, y}$ obtained from the SCHA and QMC calculations within the anisotropic superfluid (SF) phase.

In Fig. 4 we show the ratio of the renormalized to the bare helicity modulus $\gamma_{\alpha} / \gamma_{\alpha}^{0}$ as a function of the interaction strength $U / J_{x}^{0}$. The bare anisotropy ratio parameter is chosen to be $\eta_{0}=0.5$ [Fig. 4(a)] and $\eta_{0}=0.1$ [Fig. 4(b)]. Here we keep both $U$ and $T$ constant but change $J_{x}^{0}$ and $J_{y}^{0}$ in order to compare with QMC data more easily. As expected, increasing the strength of interactions, that is, increasing $U / J_{x}^{0}$, suppresses superfluidity as both $\gamma_{x}$ and $\gamma_{y}$ decrease. Note that, within the SCHA, even a weak interaction can have a strong effect on the renormalized helicity modulus $\gamma_{x, y}$. Indeed, when the interaction is larger than a critical value, the helicity modulus drops to zero in both directions discontinuously, and the system becomes a normal fluid without phase stiffness. This is a feature of the SCHA, which wrongly predicts the interaction-driven transition between the SF and the normal fluid (which at $T=0$ corresponds to the SF-to-Mott-insulator quantum phase transition) to be of first order.

In the same figure, we also show numerical results of our QMC simulation for comparison. We can see that, although the ratio of the renormalized to the bare helicity modulus obtained from QMC calculations exhibits qualitatively the same behavior as the results of the SCHA, it does not show the strong renormalization effects predicted by the SCHA at small $U / J_{x}^{0}$. Furthermore, at larger $U / J_{x}$, both $\gamma_{x} / \gamma_{x}^{0}$ and $\gamma_{y} / \gamma_{y}^{0}$ vanish rather smoothly.

In order to better understand how finite temperature and interactions influence the anisotropy ratio of the helicity modulus, we show in Figs. 5(a) and 5(b) the renormalized helicity ratio $\eta=\gamma_{y} / \gamma_{x}$ vs the bare one $\eta_{0}=\gamma_{y}^{0} / \gamma_{x}^{0}=t_{y} / t_{x}$. Results obtained from both the SCHA and QMC calculations are shown together for comparison. We see that, at low temperatures $[T / U=0.01$, Fig. 5(a)], when the system is deep in the superfluid phase, the anisotropy is barely renormalized, i.e., $\eta \simeq \eta_{0}$, and indeed our QMC results agree well with the SCHA predictions for $\eta$. Interestingly, this result also holds true at much higher temperatures [see Fig. 5(b)], except for the fact that, for small values of $\eta_{0}$, the system becomes a normal gas (i.e., the temperature used in the simulation $T / U=0.5$ is

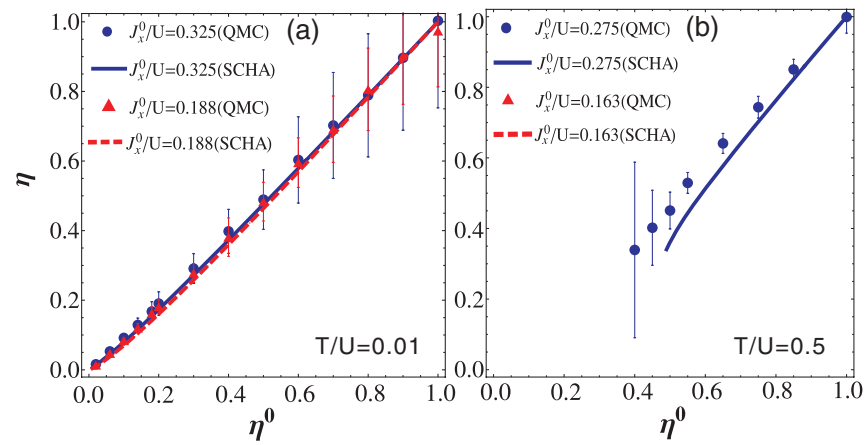

FIG. 5. (Color online) Ratio of the renormalized helicity modulus $\eta$ vs its bare (unrenormalized) value $\eta_{0}$, for (a) $T / U=0.01$ and (b) $T / U=0.5$. We choose different values of $J_{x}^{0} / U$ : (a) $J_{x}^{0} / U=$ 0.325 and $J_{x}^{0} / U=0.188$ and (b) $J_{x}^{0} / U=0.275$ and $J_{x}^{0} / U=0.163$, and vary $\eta_{0}$. Note that in (b), for $\eta_{0} \lesssim 0.5$, the temperature is higher than the BKT transition temperature and therefore both $\gamma_{x}$ and $\gamma_{y}$ vanish. We also show the results of our QMC simulations for comparison. The QMC and SCHA calculations yield results in excellent agreement, suggesting that the anisotropy ratio of the helicity modus is barely renormalized by interaction and finitetemperature effects. This is consistent with the SF phase being described by an isotropic Gaussian field theory, as discussed in Sec. II C.

larger than the BKT transition temperature $T_{c}$ for these highly anisotropic systems). This is because as the $T_{c}$ of an anisotropic superfluid $\left(\eta_{0}<1\right)$ becomes smaller, the renormalization of the helicity ratio also becomes more significant near the phase transition boundary. The agreement between SCHA and QMC results is very good.

Thus, we find that, although the SCHA and QMC calculations yield different values for the renormalized helicity moduli $\gamma_{x}$ and $\gamma_{y}$, QMC simulation shows that the renormalized anisotropy ratio $\eta=\gamma_{y} / \gamma_{x}$ is barely affected by interaction and/or temperature effects. This is consistent with the SF phase being described, in the continuum limit, by an isotropic Gaussian field theory [cf. Eq. (10) in Sec. II C], which is also correctly captured by the SCHA.

\section{B. Near the BKT transition}

In Fig. 6, we show the helicity modulus [proportional to the superfluid density, cf. Eq. (17)] as a function of temperature. The bare single-particle tunneling amplitude is $t_{x} / U=0.5$ and $t_{y} / U=0.25$, respectively, and the filling fraction is $\rho_{0} \simeq 0.63$. In Fig. $6(\mathrm{a})$, the results for the helicity moduli obtained from the SCHA and QMC calculations are compared. We thus see that, compared to the QMC results, the SCHA overestimates the temperature dependence of the helicity modulus in both directions roughly by a factor of 1.5 .

In Fig. 6(b), we show how the BKT transition temperature $T_{c}$ is determined from both the analytical results of the SCHA and the QMC data. In the case of the SCHA, we compute the phase stiffness as discussed in Sec. II C, i.e., from $K_{\beta}=$ $\beta \sqrt{J_{x} J_{y}}$, where $J_{x}$ and $J_{y}$ are solutions to the SCHA equations for given $T$ and $U, J_{x}^{0}, J_{x}^{0}$ values. Hence, $T_{c}$ is found by varying the temperature until $K_{\beta}=\frac{2}{\pi}$ (cf. Sec. II C).

As to the QMC data, $T_{c}$ is obtained as follows: As anticipated in Sec. II D, by choosing $L_{x} / L_{y}=\sqrt{t_{x} / t_{y}}$ we 

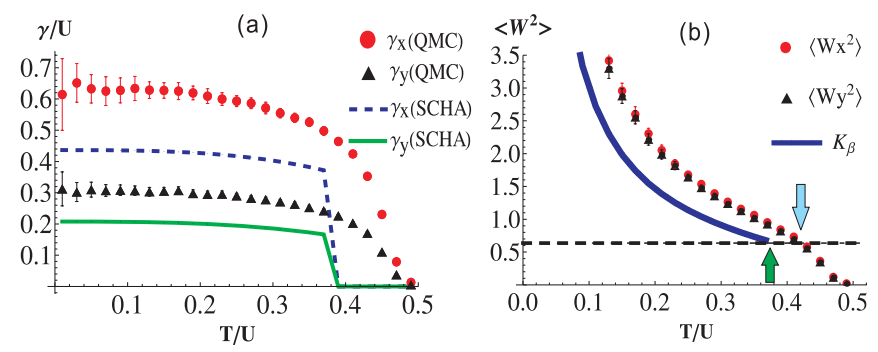

FIG. 6. (Color online) The helicity modulus as a function of temperature for a fixed interaction $U$. The bare tunneling amplitudes are $t_{x} / U=0.5, t_{y} / U=0.25$, and the density is $\rho_{0} \simeq 0.63$. (a) shows results including interaction renormalization within the SCHA, compared with the QMC results in dots and in triangles. (b) shows the winding-number fluctuation in the QMC simulations, and $K_{\beta}$ obtained analytically from the SCHA to the $X Y$ model, as a function of temperature. The horizontal dashed line indicates the universal number $2 / \pi$. The intersection of the curves (dots) and the horizontal lines gives the $T_{c}$ in the SCHA (QMC simulation), marked by arrows.

find that the winding-number fluctuations [red dots and black triangles in Fig. 6(b)] in the $x$ and $y$ directions essentially coincide. Furthermore, the $\left\langle W_{x, y}^{2}\right\rangle$ show a kink at a temperature which is essentially equal to the one obtained by requiring that $T_{c}$ (cf. Sec. II C):

$$
\begin{aligned}
K_{\beta}^{\mathrm{QMC}}\left(T_{c}\right) & =\frac{1}{T_{c}} \sqrt{\gamma_{x}\left(T_{c}\right) \gamma_{y}\left(T_{c}\right)} \\
& =\sqrt{\left\langle W_{x}^{2}\left(T_{c}\right)\right\rangle\left\langle W_{y}^{2}\left(T_{c}\right)\right\rangle} \simeq\left\langle W_{x, y}^{2}\right\rangle=\frac{2}{\pi},
\end{aligned}
$$

where Eqs. (14) and (16) have been used. In Fig. 6(b), we have indicated the universal value of $\frac{2}{\pi}$ by a horizontal line. As explained in Sec. IIC, in the SCHA, we assume that $K_{\beta}$ vanishes for for $T>T_{c}$. However, in the QMC calculations, finite-size effects round off the expected thermodynamic-limit discontinuity of $K_{\beta}^{\mathrm{QMC}}$ at $T=T_{c}$. Yet, as discussed in Sec. II D, the value of $T_{c}$ estimated from the kink in the Monte Carlo data is converged for the system sizes that we used $\left(L_{x}>100\right)$. Finally, the comparison of $T_{c}$ as obtained from QMC and SCHA calculations is shown in Fig. 8 and will be explained in more detail further below.

\section{LARGE ANISOTROPY}

\section{A. SCHA for coupled TLLs}

To begin with, let us note that, for the 1D Bose-Hubbard model, the Luttinger parameters $K$ and $v$ that determine the properties of the TLLs in the decoupled limit [cf. Eq. (6) for $g_{J}^{0}=0$ ] cannot be analytically obtained for general lattice fillings and values of $U / t_{x}$ [Eq. (1) for $t_{y}=0$ ]. Thus, in order to extract the Luttinger-liquid parameter $K$ and sound velocity $v$, we have carried out additional QMC calculations for the 1D Bose-Hubbard model to extract these parameters. Using the relations $v / K=1 / \pi \kappa$ and $v K=\pi L_{x} T\left\langle W_{x}^{2}\right\rangle$, where $\kappa=\partial \rho / \partial \mu$ is the compressibility and $\left\langle W_{x}^{2}\right\rangle$ is the winding number fluctuation along the $x$ direction for $T / U \ll 1$ [44]. In Fig. 7 the numerical Luttinger parameters $K$ and $v$ are shown as functions of the temperature $T / U$ for a large size of the $1 \mathrm{D}$ system of $L_{x}=150$. The parameters characterizing one
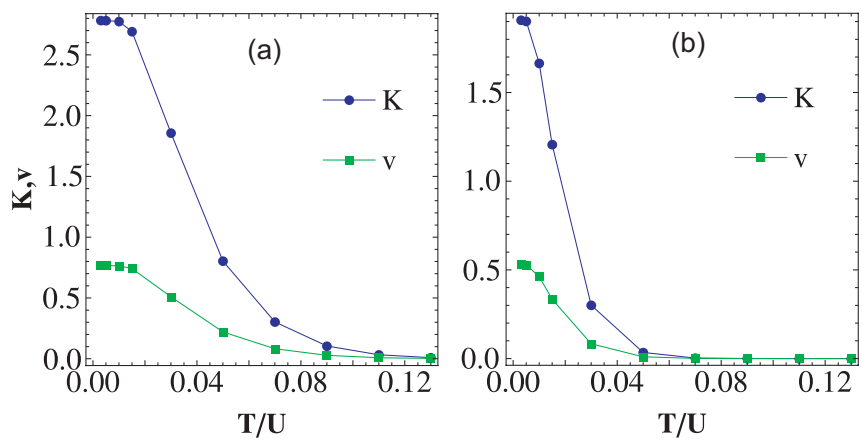

FIG. 7. (Color online) The Luttinger parameters $K$ and $v$ as a function of the temperature $T / U$. For the 1D Bose-Hubbard model, we fix $L_{x}=150$ for both plots, and (a) $t_{x} / U=0.5, t_{y} / U=0, \rho_{0} \simeq$ 0.7 and (b) $t_{x} / U=0.25, t_{y} / U=0, \rho_{0} \simeq 0.8$, respectively.

(decoupled) TLL correspond to the extrapolation of this results to very low temperature. Thus, for $T / U=0.005$ we find $K \simeq 2.77$ and $v \simeq 0.77$ for $t_{x} / U=0.5, \rho_{0} \simeq 0.7$ in Fig. 7(a), and $K \sim 1.91$, and $v \simeq 0.53$ for $t_{x} / U=0.25, \rho_{0} \simeq 0.8$ in Fig. 7(b).

Next, we describe the result of applying the SCHA to a system of coupled TLLs. Compared to the case of small anisotropy discussed above [Eq. (A12)], in this case only $J_{y}$ is renormalized, and all the interaction dependence of $J_{y}$ enters through the Luttinger parameters. However, as discussed in Sec. IIC, the system of coupled TLLs at finite temperature also belongs to the 2D $X Y$ universality class $[13,14]$. Thus, the BKT critical temperature can be found from the equation

$$
K_{\beta}=2 \frac{\sqrt{K v J_{y}\left(T_{c}\right) / 2 \pi}}{T_{c}}=\frac{2}{\pi} .
$$

In the SCHA calculations, we have chosen the shortdistance cutoff such that $K v a_{0} / 2 \pi \simeq J_{x}$, when comparing the TLL Gaussian model of Eq. (8) with the Gaussian model in Eq. (5).

In Fig. 8 (black dots), we show the BKT critical temperature $T_{c}$ computed using the SCHA and QMC methods as a function

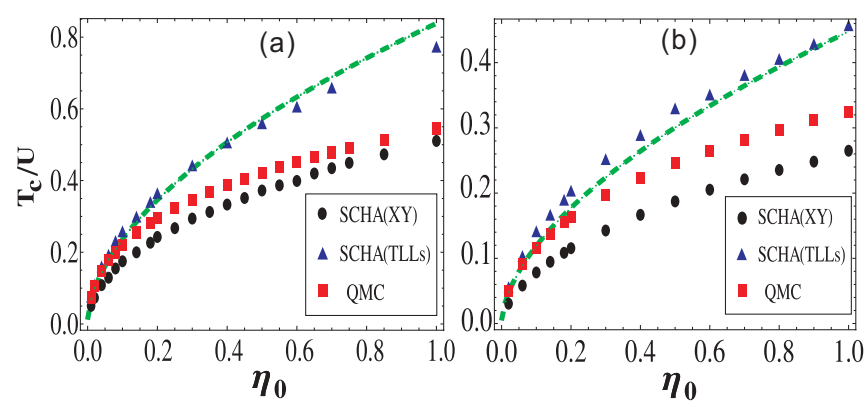

FIG. 8. (Color online) $T_{c}$ as a function of the bare anisotropy ratio $\eta_{0}=J_{y}^{0} / J_{x}^{0}=t_{y} / t_{x}$ for $t_{x} / U=0.5, \rho_{0} \simeq 0.65 \pm 0.05\left(J_{x}^{0} \simeq 0.325\right)$ (a) and $t_{x} / U=0.25, \rho_{0} \simeq 0.75 \pm 0.05\left(J_{x}^{0} / U \simeq 0.1825\right)$ (b) The blue triangles are the results obtained by the SCHA to the $X Y$ model. The black dots correspond to the results obtained by the SCHA to an array of coupled TLLs. The red squares are the QMC data. $T_{c}$ is determined by the methods discussed in Sec. III B. On both panels, the green dashed curve is a fit to the scaling behavior of $T_{c}$ with the bare anisotropy ratio $\eta_{0}$, yielding $T_{c} / U \simeq 0.837 \eta_{0}^{0.55}$ (a) and $T_{c} / U \simeq$ $0.448 \eta_{0}^{0.575}(\mathrm{~b})$. 
of the bare anisotropy ratio. As discussed above, $T_{c}$ goes to zero gradually as the bare anisotropy ratio $\eta_{0}$ becomes larger, reflecting the fact that there is no superfluid phase transition at finite temperature in a 1D system. From both panels in Fig. 8, it can be seen that the SCHA to the $X Y$ model provides a reasonably good description of $T_{c}$ (compared to the QMC results) for $\eta_{0} \approx 1$ and weak interactions [Fig. 8(a)], but it deviates from the QMC results for stronger interactions [Fig. 8(b)] and small $\eta_{0}$. On the other hand, the results obtained by applying the SCHA to an array of coupled TLLs are found to be closer to the QMC results for $T_{c}$ in the large-anisotropy regime (i.e., small $\eta_{0}$ ). These results are consistent with the expectation that the SCHA to the $X Y$ model should be more accurate in the small-anisotropy regime, whereas applying the SCHA to an array of coupled TLLs becomes a better approximation in the limit of large anisotropy.

\section{B. RG scaling for the critical temperature}

Besides the numerical calculations of the BKT critical temperature, from our QMC data we can also extract the scaling behavior of $T_{c}$ with the anisotropy ratio $\eta_{0}$. This can be compared with the results obtained by the renormalizationgroup flow of the Josephson coupling in Eq. (7), which is described by the differential equation $[13,14]$

$$
\frac{d g_{J}}{d \ell}=\left(2-\frac{1}{2 K}\right) g_{J},
$$

where the flow parameter $\ell=\ln \left[a(\ell) / a_{0}\right]=\ln [\Lambda(0) / \Lambda(\ell)]$ with $a(\ell)=a_{0} e^{\ell}$. Since $K \in[1,+\infty)$ for the Bose-Hubbard model is far from the critical point $K^{*}=1 / 4$, we can neglect the renormalization of $K$ and treat it as a constant $[13,14]$. Therefore, the solution to $(21)$ reads $g_{J}(\ell) \simeq g_{J}(0) e^{(2-1 / 2 K) \ell}$. To complete the solution, we need to recall that the bare (energy) cutoff $\Lambda(0) \approx t_{x}$, and $g_{J}(0) \simeq 2 \pi t_{y} \rho_{0} a_{0}^{2} / v$. In order to estimate the critical temperature at which the system will enter the SF phase, we note that, at finite temperatures, the $\mathrm{RG}$ flow is cut off at the scale $\Lambda(\ell) \simeq T$, and $g_{J}\left(T=T_{c}\right) \sim 1$. Hence, provided (21) provides an accurate description of the entire flow [i.e., for small enough $g_{J}(0)$ ], we have

$$
T_{c} \simeq C \eta_{0}^{1 /(2-1 / 2 K)}=C \eta_{0}{ }^{2 K /(4 K-1)},
$$

where $C$ is a prefactor that depends on microscopic details of the model, and can be obtained by fitting the above scaling law to the QMC results. It is worth noting [14] that the same scaling law for $T_{c} / t_{x}$ with $\eta_{0}$ can also be obtained using meanfield theory, i.e., by assuming that $\left\langle e^{i \theta_{n}(0)}\right\rangle=\phi_{0}(T)$. However, as discussed in the Introduction, strictly speaking, mean-field theory is inapplicable in two dimensions due to the lack of a Bose-Einstein condensate at finite temperatures.

In Fig. 8 we use the values of the Luttinger parameters obtained earlier from QMC simulations of the 1D BoseHubbard model $[K \simeq 2.77$ and $v \simeq 0.77$ in Fig. 8 (a) and $K \sim 1.91$ and $v \simeq 0.53$ in Fig. 8(b)] to fit the scaling of $T_{c}$. In particular, the value of $K$ completely determines the exponent of the scaling law [cf. Eq. (22)], and thus the only free parameter is the prefactor $C$. The fit yields $T_{c} / U \simeq 0.837 \eta_{0}^{0.55}$ for the data in Fig. 8(a) and $T_{c} / U \simeq 0.448 \eta_{0}^{0.575}$ for the data in Fig. 8(b). Using the TLL parameters obtained from $T / U=$
0.005 , the predicted $T_{c}$ values of the SCHA to TLLs are close to the results of QMC calculations in large-anisotropy regimes.

\section{CONCLUSION}

In summary, two different approaches, the SCHA and QMC methods, reveal the highly nontrivial features of the helicity modulus and the BKT phase transition in the 2D Bose-Hubbard model with anisotropic hopping. These characteristic features simulated by the QMC method using a specific system aspect ratio, $L_{x} / L_{y}=\sqrt{t_{x} / t_{y}}$, are consistent with the rescaling of the effective sine-Gordon model. We show how the interaction and finite-temperature effects influence the helicity modulus and find profound agreement of the anisotropy of the helicity modulus given by the SCHA and QMC methods. As we drive the system towards the extremely anisotropic limit, the BKT transition temperature approaches absolute zero and the transition thus becomes a $(1+1) X Y$ quantum critical point at the end of a line of classical $2 \mathrm{D} X Y$ critical points. In particular, through the RG scheme for coupled TLLs, we obtain the scaling relation of $T_{c}$ with the anisotropy ratio. Employing ultracold atoms in a controllable optical lattice opens avenues to confirm our results for the 2D anisotropic Bose-Hubbard model.

\section{ACKNOWLEDGMENTS}

This work is supported by NSC grants and also NCTS. M.A.C. gratefully acknowledges the hospitality of NCTS (Taiwan) and the financial support of the Spanish MEC through Grant No. FIS2010-19609-C02-02.

\section{APPENDIX A: SELF-CONSISTENT HARMONIC APPROXIMATION}

To find the optimally quadratic approximation to the $X Y$ model, we employ the self-consistent harmonic approximation. In this approach, the action of the $X Y$ or quantum rotor model, Eq. (2), is approximated by an anisotropic Gaussian model:

$$
\begin{aligned}
S_{G}[\theta] & =\int_{0}^{\beta} d \tau\left\{\sum_{i} \frac{\left(\partial_{\tau} \theta_{i}\right)^{2}}{2 U}+\sum_{\langle i, j\rangle} J_{i j}\left(\theta_{i}-\theta_{j}\right)^{2}\right\} \\
& =\frac{1}{2} \sum_{\mathbf{k}, \omega_{n}} G_{v}^{-1}\left(\mathbf{k}, \omega_{n}\right)\left|\theta\left(\mathbf{k}, \omega_{n}\right)\right|^{2},
\end{aligned}
$$

where $\omega_{n}=2 \pi T n$, and the single-particle Green's function is given by

$$
G_{v}^{-1}\left(\mathbf{k}, \omega_{n}\right)=\frac{\omega_{n}^{2}}{U}+\sum_{\alpha} 8 J_{\alpha} \sin ^{2}\left(k_{\alpha} \hat{a}_{\alpha} / 2\right) .
$$

Next, we make use of Feynman's variational principle, which states that

$$
F=-\frac{1}{\beta} \ln Z \leqslant \tilde{F}\left[G_{v}\right]=F_{v}+\frac{1}{\beta}\left\langle S[\theta]-S_{G}[\theta]\right\rangle_{v},
$$

where \langle\rangle$_{v}$ denotes the average with respect to $S_{G}[\theta]$ and $S$ is the $X Y$ model action. Since

$$
e^{-\beta F_{v}}=\int D \theta e^{-S_{v}[\theta]}=\prod_{\mathbf{k}, \omega_{n}} G_{v}\left(\mathbf{k}, \omega_{n}\right)^{-1 / 2},
$$


the first term of $\tilde{F}\left[G_{v}\right]$ is

$$
F_{v}=-\frac{1}{2 \beta} \sum_{\mathbf{k}, \omega_{n}} \ln G_{v}\left(\mathbf{k}, \omega_{n}\right) .
$$

The remaining contributions to $\tilde{F}\left[G_{v}\right]$ are

$$
\begin{aligned}
\left\langle S_{X Y}[\theta]-S_{G}[\theta]\right\rangle_{v} & \left\langle\int_{0}^{\beta} d \tau\left\{\sum_{i} \frac{1}{2 U}\left(\partial_{\tau} \theta_{i}\right)^{2}-\sum_{\langle i, j\rangle} 2 J_{i j}^{0} \cos \left(\theta_{i}-\theta_{j}\right)\right\}\right\rangle_{v} \\
& -\left\langle S_{G}[\theta]\right\rangle_{v} \\
= & \sum_{\mathbf{k}, \omega_{n}} \frac{\omega_{n}^{2}}{2 U} G_{v}\left(\mathbf{k}, \omega_{n}\right)+\left\langle S_{\cos }\right\rangle_{v}-\text { const }
\end{aligned}
$$

with

$$
\left\langle S_{\cos }\right\rangle_{v}=-\int_{0}^{\beta} d \tau \sum_{\langle i, j\rangle} 2 J_{i j}^{0}\left\langle\cos \left(\theta_{i}-\theta_{j}\right)\right\rangle_{v} .
$$

Hence,

$$
\begin{aligned}
\left\langle\cos \left(\theta_{i}-\theta_{j}\right)\right\rangle_{v} & =\operatorname{Re}\left[e^{-(1 / 2)\left\langle\left(\theta_{i}-\theta_{j}\right)^{2}\right\rangle_{v}}\right] \\
& =\operatorname{Re}\left[{ }^{G_{v}\left(r_{i}-r_{j}, 0\right)-G_{v}(0,0)}\right]
\end{aligned}
$$

by the cumulant expansion. Here

$$
G_{v}(r, \tau)=\frac{1}{\beta \Omega} \sum_{\mathbf{k}, \omega_{n}} e^{i \mathbf{k} r} e^{-i \omega_{n} \tau} G_{v}\left(\omega_{n}, \mathbf{k}\right)
$$

is the single-particle Green's function in real space. Therefore we have

$$
\begin{aligned}
\left\langle S_{\cos }\right\rangle_{v} & =-\int_{0}^{\beta} d \tau \sum_{\langle i, j\rangle} 2 J_{i j}^{0} \operatorname{Re}\left[e^{G_{v}\left(r_{i}-r_{j}, 0\right)-G_{v}(0,0)}\right] \\
& =-\beta \sum_{i} \sum_{\mathbf{t}=\hat{a}_{x}, \hat{a}_{y}} 2 J_{\mathbf{t}}^{0} \operatorname{Re}\left[e^{G_{v}(\mathbf{t}, 0)-G_{v}(0,0)}\right] \\
& =-\beta \Omega \sum_{\alpha} 2 J_{\alpha}^{0} \operatorname{Re}\left[e^{(1 / \Omega \beta) \sum_{\mathbf{k}, \omega_{n}}\left(e^{i \mathbf{k} \cdot \hat{a}_{\alpha}}-1\right) G_{v}\left(\mathbf{k}, \omega_{n}\right)}\right] .
\end{aligned}
$$

Upon combining the above results and finding the extrema of $\tilde{F}\left[G_{v}\right]$, i.e.,

$$
\frac{\delta F^{\prime}\left[G_{v}\right]}{\delta G_{v}\left(\mathbf{k}, \omega_{n}\right)}=0
$$

we find

$$
\begin{aligned}
& \frac{1}{G_{v}\left(\mathbf{q}, \omega_{n}\right)} \\
& =\frac{\omega_{n}^{2}}{U}+8 \sum_{\alpha} J_{\alpha}^{0} \sin ^{2}\left(q_{\alpha} \hat{a}_{\alpha} / 2\right) e^{(1 / \Omega \beta) \sum_{\mathbf{k}, \omega_{n}}\left(e^{i \mathbf{k} \hat{a}_{\alpha}}-1\right) G_{v}\left(\mathbf{k}, \omega_{n}\right)} \\
& \quad \equiv \frac{\omega_{n}^{2}}{U}+8 \sum_{\alpha} J_{\alpha} \sin ^{2}\left(q_{\alpha} \hat{a}_{\alpha} / 2\right) .
\end{aligned}
$$

Using the Matsubara sum

$$
\begin{aligned}
\frac{1}{\beta} \sum_{\omega_{n}} G_{v}\left(\mathbf{k}, \omega_{n}\right) & =\frac{U}{\beta} \sum_{\omega_{n}} \frac{1}{\omega_{n}^{2}+\omega_{\mathbf{k}}^{2}} \\
& =\frac{U}{2 \omega_{\mathbf{k}}} \operatorname{coth}\left(\frac{\beta \omega_{\mathbf{k}}}{2}\right),
\end{aligned}
$$

we conclude that

$$
\ln \frac{J_{\alpha}}{J_{\alpha}^{0}}=\frac{1}{\Omega} \sum_{\mathbf{k}}\left(e^{i \mathbf{k} \cdot \hat{a}_{\alpha}}-1\right) \frac{U}{2 \omega_{\mathbf{k}}} \operatorname{coth}\left(\frac{\beta \omega_{\mathbf{k}}}{2}\right) .
$$

Note that $\omega_{\mathbf{k}}=2 \sqrt{2 U} \sqrt{J_{x} \sin ^{2}\left(k_{x} a / 2\right)+J_{y} \sin ^{2}\left(k_{y} a / 2\right)}$ is the phonon (Bogoliubov) excitation energy.

\section{APPENDIX B: THE SCHA FOR COUPLED TLLS}

Applying the methods of the previous section to the action of Eq. (7), the following equation for the renormalized parameter $J_{y}\left(=g_{J} v / \pi a_{0}^{2}\right)$ is obtained:

$$
\ln \frac{g_{J}}{g_{J}^{0}}=\frac{v K^{-1}}{L_{x} L_{y}} \sum_{\mathbf{k}} \frac{e^{i \mathbf{k} \cdot \hat{\mathbf{y}}}-1}{\omega_{\mathbf{k}}} \operatorname{coth}\left(\frac{\beta \omega_{\mathbf{k}}}{2}\right),
$$

where $\omega_{\mathbf{k}}=2 \sqrt{v^{2}\left(k_{x} / 2\right)^{2}+\frac{2 \pi v J_{y}}{K} \sin ^{2}\left(k_{y} / 2\right)}$.
[1] M. Greiner, O. Mandel, T. Esslinger, T. W. Hansch, and I. Bloch, Nature (London) 415, 39 (2002).

[2] D. Jaksch, C. Bruder, J. I. Cirac, C. W. Gardiner, and P. Zoller, Phys. Rev. Lett. 81, 3108 (1998).

[3] I. Bloch, J. Dalibard, and W. Zwerger, Rev. Mod. Phys. 80, 885 (2008).

[4] T. Stöferle, Henning Moritz, Christian Schori, Michael Köhl, and Tilman Esslinger, Phys. Rev. Lett. 92, 130403 (2004).

[5] Chen-Lung Hung, Xibo Zhang, Nathan Gemelke, and Cheng Chin, Nature (London) 470, 236 (2011).

[6] S. Trotzky, L. Pollet, U. Schnorrberger, F. Gerbier, I. Bloch, N. V. Prokof'ev, B. Svistunov, and M. Troyer, Nat. Phys. 6, 998 (2010).

[7] X. Zhang, C.-L. Hung, S.-K. Tung, and C. Chin, Science 335, 1070 (2012).
[8] E. Haller et al., Nature (London) 466, 597 (2010).

[9] C. Becker et al., New J. Phys. 12, 065025 (2010).

[10] Z. Hadzibabic, P. Kruger, M. Cheneau, B. Battelier, and J. Dalibard, Nature (London) 441, 1118 (2006).

[11] M. A. Cazalilla, A. Iucci, and T. Giamarchi, Phys. Rev. A 75, 051603(R) (2007).

[12] L. M. Mathey, A. Polkovnikov, and A. H. Castro-Neto, Europhys. Lett. 81, 10008 (2008).

[13] A. F. Ho, M. A. Cazalilla, and T. Giamarchi, Phys. Rev. Lett. 92, 130405 (2004).

[14] M. A. Cazalilla, A. F. Ho, and T. Giamarchi, New J. Phys. 8, 158 (2006).

[15] V. Cataudella and P. Minnaghen, Physica C 166, 442 (1990).

[16] B. Chattopadhyay and S. R. Shenoy, Phys. Rev. Lett. 72, 400 (1994). 
[17] P. Minnhagen and P. Olsson, Phys. Rev. B 44, 4503 (1991).

[18] L. Benfatto, C. Castellani, and T. Giamarchi, Phys. Rev. Lett. 98, 117008 (2007).

[19] O. A. Starykh and L. Balents, Phys. Rev. Lett. 98, 077205 (2007).

[20] M. Kohno, O. A. Starykh, and L. Balents, Nat. Phys. 3, 790 (2007).

[21] T. Giamarchi, Ch. Regg, and O. Tchernyshyov, Nat. Phys. 4, 198 (2008).

[22] Ch. Rüegg et al., Phys. Rev. Lett. 101, 247202 (2008); P. Bouillot et al., Phys. Rev. B 83, 054407 (2011).

[23] W. P. Su, J. R. Schrieffer, and A. J. Heeger, Phys. Rev. Lett. 42, 1698 (1979); D. C. Tsui, H. L. Störmer, and A. C. Gossard, ibid. 48, 1559 (1982); R. B. Laughlin, ibid. 50, 1395 (1983); D. Arovas, J. R. Schrieffer, and F. Wilczek, ibid. 53, 722 (1984).

[24] M. A. Cazalilla, R. Citro, T. Giamarchi, E. Orignac, and M. Rigol, Rev. Mod. Phys. 83, 1405 (2011).

[25] J. M. Kosterlitz and D. J. Thouless, J. Phys. C 6, 1181 (1973).

[26] D. J. Bishop and J. D. Reppy, Phys. Rev. Lett. 40, 1727 (1978).

[27] D. M. Gangardt, P. Pedri, L. Santos, and G. V. Shlyapnikov, Phys. Rev. Lett. 96, 040403 (2006).

[28] S. Bergkvist, A. Rosengren, R. Saers, E. Lundh, M. Rehn, and A. Kastberg, Phys. Rev. Lett. 99, 110401 (2007); M. Rehn et al., Eur. Phys. J. D 49, 223 (2008).

[29] J. K. Freericks, Phys. Rev. A 78, 013624 (2008).

[30] J. Taniguchi, Y. Aoki, and M. Suzuki, Phys. Rev. B 82, 104509 (2010); J. Taniguchi, R. Fujii, and M. Suzuki, ibid. 84, 134511 (2011).
[31] D. R. Nelson and J. M. Kosterlitz, Phys. Rev. Lett. 39, 1201 (1977).

[32] T. Giamarchi and B. S. Shastry, Phys. Rev. B 51, 10915 (1995).

[33] A. Del Maestro and I. Affleck, Phys. Rev. B 82, 060515(R) (2010).

[34] T. Eggel, M. A. Cazalilla, and M. Oshikawa, Phys. Rev. Lett. 107, 275302 (2011).

[35] R. P. Feynman, Statistical Mechanics (Benjamin, Reading, MA, 1972).

[36] J. V. Jose, Leo P. Kadanoff, Scott Kirkpatrick, and David R. Nelson, Phys. Rev. B 16, 1217 (1977).

[37] Naoto Nagaosa, Quantum Field Theory in Condensed Matter Physics (Springer, Heidelberg, Germany, 1999).

[38] L. Pollet, K. Van Houcke, and S. M. A. Rombouts, J. Comput. Phys. 225, 2249 (2007).

[39] Barbara Capogrosso-Sansone, Şebnem Güneş Soÿler, Nikolay Prokof'ev, and Boris Svistunov, Phys. Rev. A 77, 015602 (2008).

[40] Lode Pollet, Corinna Kollath, Kris Van Houcke, and Matthias Troyer, New J. Phys. 10, 065001 (2008).

[41] N. V. Prokof'ev and B. V. Svistunov, Phys. Rev. B 61, 11282 (2000).

[42] Michael E. Fisher, Michael N. Barber, and David Jasnow, Phys. Rev. A 8, 1111 (1973).

[43] E. L. Pollock and D. M. Ceperley, Phys. Rev. B 36, 8343 (1987).

[44] M. A. Cazalilla, J. Phys. B 37, S1 (2004). 